\title{
Traduire
}

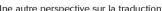

Revue française de la traduction

$226 \mid 2012$

Face au miroir

\section{Traduire la nouvelle génération d'écrivains égyptiens : réussir un puzzle minimaliste}

\section{Sahar Samir Youssef}

\section{(2) OpenEdition}

1 Journals

Édition électronique

URL : http://journals.openedition.org/traduire/167

DOI : $10.4000 /$ traduire.167

ISSN : 2272-9992

Éditeur

Société française des traducteurs

Édition imprimée

Date de publication : 1 janvier 2012

ISBN : 039-773X

ISSN : 0395-773X

\section{Référence électronique}

Sahar Samir Youssef, «Traduire la nouvelle génération d'écrivains égyptiens : réussir un puzzle minimaliste », Traduire [En ligne], 226 | 2012, mis en ligne le 28 janvier 2014, consulté le 09 octobre 2020. URL : http://journals.openedition.org/traduire/167 ; DOI : https://doi.org/10.4000/traduire.167 


\title{
Traduire la nouvelle génération d'écrivains égyptiens : réussir un puzzle minimaliste
}

\author{
Sahar Samir Youssef
}

1 Il ne fait aucun doute que le paysage littéraire égyptien a énormément changé pendant les dernières décennies. Et ce changement a été encore accentué par la tendance internationale; un certain nombre de mouvements littéraires modernes (Futurisme, Objectivisme, Existentialisme, Imagisme, Lettrisme, Nouveau roman, etc.) ont de fait largement influencé des générations d'écrivains égyptiens en laissant des traces dans les différentes formes de leur production créative. Et, bien que la plupart de ces mouvements trouvent leur origine dans la littérature européenne, les auteurs égyptiens ont, avec le temps, su dépasser la simple imitation et donné naissance à une authentique littérature égyptienne. Les écrits de certains d'entre eux ont même connu un retentissement qui leur a valu des traductions dans une ou plusieurs langues, et se font ainsi remarquer sur la scène internationale.

Dans le cadre d'une recherche plus vaste sur la possibilité de traduire la nouvelle littérature égyptienne et les conditions de réalisation de ce type de traduction, nous nous interrogerons, dans la présente étude, sur l'originalité d'un courant qui, venant après le Nouveau Roman, a réinventé la fiction et présente un nouvel art d'écrire, à savoir le Minimalisme.

\section{Qu'est-ce que le Minimalisme?}

3 La définition du Minimalisme s'articule autour de sa conception comme identification de l'art avec ses rudiments (principe d'économie maximale des moyens). Il relève d'une tentative de réduction systématique d'une pratique à ses composantes ultimes et essentielles. Largement développée dans les différents arts comme la peinture, l'architecture, le design et même la musique, l'esthétique minimaliste a eu peu à peu une influence considérable sur le monde des lettres. 
4 Le Minimalisme littéraire a vu le jour aux États-Unis, au milieu des années soixante, en réaction au débordement subjectif de l'Expressionnisme et aux métafictions postmodernes.

5 Abandonnant les excès du Postmodernisme, les auteurs minimalistes souhaitaient revenir à des formes de récit moins bavardes et plus épurées. Le principe de base de ce nouveau régime d'écriture fictionnelle est simple: moins écrire, de façon concise et sobre, pour exprimer plus, sous-entendre beaucoup et mieux mettre en valeur.

Le Minimalisme littéraire se définit aussi par l'hyperprécision de la représentation du banal, par une attention accrue portée aux éléments les plus basiques, aux objets et aux détails de la vie quotidienne. Une réduplication exacerbée de la réalité contemporaine ayant pour centre la souffrance et la complexité de l'être humain.

7 Le Minimalisme semble en effet inspirer de plus en plus d'écrivains. Des milliers de jeunes romanciers se ruent chaque année dans les ateliers de Creative Writing des universités amé- ricaines en vue d'y entreprendre une carrière littéraire.

Dans la nouvelle littérature égyptienne, un roman se distingue par son caractère novateur et anti-conformiste : Dans la peau de 'Abbas El 'Abd. Déroutant et déstabilisant, ce roman devient vite l'exemple le plus retentissant d'une nouvelle écriture minimaliste en Égypte. Et son auteur, Ahmad Alaidy, est considéré comme le plus illustre représentant du Minimalisme de sa génération.

\section{« Dans la peau de 'Abbas El 'Abd » d'Ahmad Alaidy}

Égyptien, né en 1974 en Arabie Saoudite, Ahmad Alaidy est un artiste talentueux à la création artistique extrêmement polyvalente. Après des études de marketing à l'Université du Caire, il exerce dans plusieurs domaines : journaliste, rédacteur de quizz pour la télévision, graphiste et designer de livres pour la jeunesse, scénariste de bandes dessinées et de comics, cinéaste et poète.

Attiré par le Minimalisme, Alaidy suit une formation en ligne centrée sur ses techniques scripturales dans l'atelier du célèbre romancier et nouvelliste américain Chuck Palahniuk, considéré comme le plus minimaliste des écrivains américains contemporains.

11 Dans la peau de 'Abbas El 'Abd est le premier roman d'Alaidy. Publié au Caire en $2003 \mathrm{chez}$ Merit sous le titre An takoun Abbas $E l \mathrm{Abd}$, le roman connut dès sa parution un succès retentissant. S'affirmait ainsi, avec Alaidy et son roman très contemporain un nouveau courant : le Minimalisme positif opposé à la narration traditionnelle.

L'engouement pour ce roman d'une brûlante actualité - devenu le roman culte de toute une génération, et qui a valu à son auteur le prix du meilleur roman égyptien moderne, décerné par la fondation Sawiris en 2006, passa les frontières. Il est traduit dans plusieurs langues: anglais, allemand, italien, polonais et turc. En France, une traduction, réalisée par Khaled Osman, est publiée chez Actes Sud en 2010.

13 Le caractère aliénant de la situation de l'individu contemporain constitue le thème central du roman: violence entre humains, communication et entente difficiles. Le texte décrit le malaise, l'existence tourmentée et l'égarement de toute une jeunesse frustrée car parvenue à l'âge adulte au moment où son pays n'a plus d'idéal politique. Dans une démarche très audacieuse, Alaidy enfonce ce monde secret des jeunes, écrasés 
par le chômage et pris dans les rouages de l'ennui, la misère, la mondialisation, la consommation et nombre d'autres problèmes qui les poussent à devenir eux-mêmes de simples objets de consommation. Livrés à eux-mêmes, ils se forgent un monde parallèle en s'enfermant dans l'univers des nouvelles technologies: Internet et la téléphonie mobile, au point de devenir "esclaves asservis à l'empire de Bill Gates", tel que se décrit le personnage principal dans les premières pages du roman.

C'est à travers les aventures étranges, pleines d'absurdité, d'incertitude et d'anxiété d'un jeune homme schizophrène qu'Alaidy nous dépeint avec génie sa perception minimaliste du quotidien. La psyché du personnage se tord et se distord en essayant de s'improviser un nouvel équilibre personnel et social. Pour ce faire, il recourt aux thérapies de groupe. Déboussolé et élevé par un oncle psychiatre, complètement déjanté lui-aussi, il est initié à la vie par un ami anarchiste, un collectionneur de queues de lézards qui s'appelle Abbas El Abd. Ce dernier lui propose de vivre dans son appartement et se fixe comme objectif de l'éduquer en l'initiant aux règles de sa propre philosophie qui l'aideraient, selon lui, à perfectionner (" upgrade ») sa vision et remettre à jour ("update») son vécu. Sur cela, ses farces inondent le texte. Pour sortir son ami de son isolement, Abbas lui organise une rencontre avec deux filles qui portent le même prénom, au même endroit et en même temps, tout en se faisant passer pour lui, ce qui donne lieu à une situation drôle, pleine de quiproquos.

C'est à travers la peinture de ces moments les plus anodins qu'Alaidy nous convie à un joli moment d'introspection et de contemplation. C'est en nous renvoyant le reflet de notre être mutilé dans ses élans, bafoué dans sa mémoire que le roman nous parle de notre impuissance et de nos impostures. Bref, c'est d'une réflexion décapante sur la société égyptienne contemporaine qu'il s'agit. Nous partageons à ce propos l'opinion de Robert Solé qui écrit dans Le Monde: "C'est la schizophrénie de la Société égyptienne, tiraillée entre religion et libération, qu'illustre ce roman inventif et déjanté. »

\section{Un Minimalisme à l'égyptienne}

16 Le modèle minimaliste - avec sa forme non linéaire et éclatée - s'avère être le plus approprié pour exprimer une réelle souffrance et ne retenir de la réalité éprouvée que l'infime significatif.

17 Au plan de la forme, les textes appartenant à ce courant se présentent souvent sous la forme de romans et de nouvelles brèves. La narration est décousue et non événementielle, l'écriture déstructurée, et le récit fragmentaire. Les fins sont souvent arbitraires et ouvertes.

$18 \mathrm{Au}$ plan du contenu, les textes minimalistes se caractérisent par une économie d'écriture maximale (abandon des adjectifs, adverbes et longues descriptions vidées de sens). Se contentant de décrire sèchement ce qui est immédiatement perceptible, ils contiennent peu de langage figuratif. Le style minimaliste consiste à utiliser un champ lexical simple, se servir de tournures abordables, choisir des mots courants, familiers voire vulgaires, dans le but de marquer son lecteur.

19 La formation aux techniques minimalistes qu'a reçue Alaidy et sa forte imprégnation par l'esprit de la société égyptienne lui ont permis de jouer sur les codes esthétiques du Minimalisme, en y mélangeant toute la sève populaire, sans pour autant leur faire perdre leur spécificité. Il introduit des aspects de la littérature égyptienne actuellement 
en vogue (neutralité du regard, fréquence de l'ironie voire du cynisme) et adopte un rythme rapide empruntant sa cadence à celle des pages web, un langage novateur. Il fait subtilement usage d'une langue inspirée des nouveaux outils de communication pour raconter une jeunesse aux mœurs ridiculement américanisées, tout en faisant état de cette terrible contamination linguistique dont souffre l'arabe du XXI ${ }^{e}$ siècle.

An takoun 'Abbas El 'Abd a été traduit par Khaled Osman. Également critique d'œuvres littéraires et cinématographiques, et tout récemment romancier, Khaled Osman est l'un des rares traducteurs littéraires confirmés de notre temps. Il traduit essentiellement des auteurs égyptiens contemporains dont notamment Naguib Mahfouz et Gamal Ghitany. On lui doit aussi de célèbres traductions d'importants auteurs arabes, comme la romancière palestinienne Sahar Khalifa, l'irakienne Inaam Kachachi et tout récemment, le yéménite Ali al-Muqri. Sa carrière de traducteur débute en 1984 où, - à la fois passionné par la littérature arabe en général et égyptienne en particulier, et déçu par la pauvreté de la bibliothèque arabe traduite en français - il entame sa première traduction parue un an après chez Sindbad: Le voleur et les chiens de Mahfouz qui lui valut, avec la traduction de Récits de notre quartier, le prix de l'Académie Française en 1988. Osman a été plusieurs fois récompensé pour le sérieux et la qualité de son travail : il a reçu le Prix du Roman Arabe, le Prix des Lecteurs du Var, le Prix Laure Bataillon et le Prix Amédée Pichot.

\section{Traduire ou rapiécer le puzzle minimaliste : une approche dynamique}

Dans la peau de 'Abbas $\mathrm{El}$ 'Abd semble être un terrain d'investigation en traduction assez privilégié, tant par la richesse de ses composantes que par la délicatesse de leur imbrication. Il s'agit d'un système ouvert et dynamique, mettant en œuvre des combinaisons stylistiques nouvelles sollicitant la créativité du traducteur. Des contraintes d'une nature spécifique s'imposent, en ce sens que le texte se présente comme une entité complexe : écriture, image, son. Les problèmes pour le traducteur se répartissent alors sur deux niveaux: le niveau linguistique et le niveau extralinguistique.

En effet, la traduction des phénomènes linguistiques dont use Alaidy et de tous les autres éléments suscitant un effet revêt une grande importance pour le respect de l'esprit du texte original. Les théoriciens de la traduction n'ont de cesse de rappeler la nécessité de la transmission des effets stylistiques que certains qualifient de "plaisir esthétique de l'original », ou mieux encore de «ta'am » ${ }^{1}$ goût du texte, pour employer les termes de Meschonnic.

Tout contribue à faire du texte d'Alaidy un creuset regorgeant d'effets littéraires : tout d'abord, ceci s'inscrit dans l'essence et la logique même du Minimalisme qui veut que chacun de ses textes soit un vrai condensé émotif. Ensuite, il y a les tonalités que l'auteur confère à son discours : comique (à l'aide des jeux de mots, des quiproquos, etc.), philosophique (réflexions, adages et aphorismes) et ironique. Puis, l'emploi d'un matériel lexical chargé d'un degré élevé de familiarité (clichés, stéréotypes et expressions figées) et rappelant des valeurs communes garantissant l'adhésion du lecteur. Bref, des choix esthétiques qui jouent clairement sur l'effet obtenu. La 
traduction aussi est avant tout une affaire de choix. Alors, faire croiser les deux choix serait-il chose aisée?

Pour ce faire, et afin de pouvoir rapiécer ce grand puzzle minimaliste, tout en maintenant l'équilibre entre original et traduction, Khaled Osman opte la plupart du temps pour ce que Eugène Nida appelle «l'équivalence dynamique». C'est en s'opposant à toute forme de traduction littérale qui ne prendrait pas en compte la culture du lecteur que Nida propose sa théorie « d'équivalence dynamique $»^{2}$. Notion centrale dans l'œuvre de ce grand traductologue, elle s'oppose à un autre type d'équivalence: l'équivalence formelle, qui accorde une importance primordiale à la forme du texte source. Or, l'équivalence dynamique, dont Nida est partisan, vise à exprimer de la façon la plus naturelle possible le message en prenant en compte la culture du destinataire de la traduction. Elle cherche à produire chez le lecteur du texte traduit un effet équivalent à celui produit chez le lecteur du texte original :

They (the readers) are not so much concerned with the formal resemblance as with the dynamic equivalence. In other words, they want to be able to interpret the relevance of the message within the context of their own lives, without having to consider or be distracted by the formal structures of the original communicative setting. ${ }^{3}$

Estimant qu'il ne saurait y avoir de correspondance absolue entre les langues, et persuadé en même temps de la prévalence des facteurs socioculturels dans tout processus de traduction, Nida préconise l'équivalence dynamique qui consiste à trouver - par des procédés similaires - certains parallèles culturels qui rendront le message significatif dans le milieu des destinataires du texte, et produiront un impact émotionnel du même ordre.

\section{Le matériel lexical}

\section{1) Sociolecte : oralité et vulgarité}

L'esthétique du texte de Alaidy nous paraît particulièrement intéressante car travaillant essentiellement la langue, et, plus précisément, une variété non standard de la langue arabe. Jamais, à notre connaissance, une telle fidélité à la langue de la rue n'a été, à ce point, mise en exergue. On y trouve des formes méprisées et rejetées par la norme, des éclats de vulgarité, des tics de langage et des expressions familières toutes faites. Dans Dans la peau de 'Abbas El'Abd, l'oralité est omniprésente sous des formes diverses et variées. Le lexique est celui de l'arabe parlé. Et outre l'emploi du vocabulaire familier, l'auteur ponctue allègrement les propos de ses personnages de termes grossiers. Il donne essentiellement la parole à une couche spécifique du peuple (chauffeurs, soldats, serveurs de cafés, vendeurs...) utilisant ainsi une langue non conventionnelle teintée de beaucoup de vulgarité.

Dans ce roman, l'oralité revêt une fonction expressive doublée d'un marqueur d'identité sociale, ce qui rend son transfert en langue d'arrivée inéluctable. Nida ne rappelle-t-il pas que pour rendre la dimension expressive d'un message, le traducteur doit se préoccuper des facteurs sociologiques, des niveaux de langues et du rôle que ceux-ci sont appelés à jouer dans un texte?

L'équivalence dynamique aide à dégager le message des contraintes formelles pour qu'il reste identique par la réaction qu'il suscite et non par son apparence extérieure. L'approche de Khaled Osman s'inscrit dans cette logique. Comme le texte original, la 
traduction qu'il nous offre est marquée par la prédominance d'un sociolecte. L'égyptien familier n'est pas rendu pour lui- même mais pour la charge sociolectale qu'il représente. Ainsi, Osman penche vers une traduction qui naturalise et francise le langage insolite d'Alaidy.

Osman opte pour un niveau de langue familier et relâché en français. Il s'inspire librement du parler des jeunes de banlieues et de la cité en France pour recréer avec succès une langue populaire, courante et vulgaire par endroits.

Pour rendre l'oralité conférée au texte de départ, le traducteur n'hésite pas à exploiter les procédés de création argotique. Ainsi, les troncations de tous types sont courantes dans la traduction :

31 Premièrement, la troncation des négations est utilisée pour retranscrire la prononciation relâchée des personnages. L'effacement de l'adverbe "ne » dans les phrases négatives est pratiqué dans la quasi-totalité du texte: «c'est pas mal élevé de manquer de respect aux clients sous mon nez!» (p. 21), «vous comprenez rien ou quoi » (p. 35), « Tu voudrais pas qu'on aille à l'étage ?» (p. 61), « T'énerve pas. » (p. 94), «Te mets pas dans cet état. » (p. 147).

Deuxièmement, la retranscription phonétique de la prononciation relâchée propre à l'oral passe par l'élision de certaines voyelles ou l'écrasement des consonnes signalées par l'apostrophe dans la langue française :

P't - être, m'sieurs, tax...! J'suis là, m'enfin, je m'sente, eh ben, j'le referai plus, d'mais.

Sans mentionner les exemples avec les pronoms personnels (je) et (tu) qui inondent le texte. On note également dans la traduction d'Osman, l'utilisation de quelques graphies fantaisistes faisant ressortir, par la déviation qu'elles provoquent, davantage de relâchement dans la prononciation des personnages: Ouais (oui) et Nan (non). On trouve aussi : quoâââââ (quoi), kékchose (quelque chose), keskeces'ra (qu'est-ce que ce sera).

D'autres éléments aussi subissent des modifications orthographiques: certains pronoms personnels comme (il) souvent transformé en (Y), ou encore (vous) remplacé par un (Z) imitant la liaison «z'avez qu'à l'allumer.», "pourquoi z'en faites une montagne?».

Les substantifs subissent des modifications, et font l'objet d'une écriture volontairement erronée visant à retransmettre une certaine vulgarité caractérisant le langage du locuteur.: «Moi, j'saurais-t-y pas conduire? Je m'en vas te dire un truc, gamin. » (p. 22), «Mon paça! [Il veut dire mon pacha] » (p. 39), « Oh ... la pôôôôôvre » (p. 67).

Par ailleurs, on remarque également l'utilisation de mots ayant subi une resuffixation avec quelques finales caractéristiques du vocabulaire argotique comme (-0, -esque, ard, -ouiller) : mélo, frérot, courtaud, matos, soldatesque, rencard, vieillard, coquillard, farfouiller, se dépatouiller, se débarbouiller.

D'autres techniques ou phénomènes du français parlé sont employés par Osman dans sa traduction quand cela est possible : la réduplication (Tintin, pan pan, cucul), l'emploi des formes emphatiques exprimant la négation comme (nada) et (que dalle), ainsi que des termes empruntés qui alimentent le langage des jeunes tels que : (illico), (presto), et (fissa). 

non seulement le sociolecte (porteur de valeurs socioculturelles) mais aussi l'idiolecte (en cas de présence, bien entendu). En principe, les linguistes, et tout particulièrement les sociolinguistes, affirment qu'il existe toujours des zones de contact entre dialecte, sociolecte et idiolecte. Si le sociolecte est porteur de valeurs collectives, l'idiolecte procédé de codification de premier ordre - est porteur d'une vision individuelle. Chaque idiolecte possède ses formes préférées, sa phraséologie spécifique et même ses propres mots. Alaidy a plus d'une raison pour privilégier l'emploi d'idiolectes Il s'agit d'une forme laconique en parfaite harmonie avec les deux principes de base du Minimalisme : brièveté et concision. L'arme secrète du Minimalisme étant la collusion entre roman et philosophie, Alaidy trouve alors dans l'idiolecte un moyen réussi pour décrire la bataille de ses personnages contre ce monde fou, tout en n'ayant comme arme que quelques commentaires philosophiques et une liste de recommandations et de conseils échangés entre eux. C'est la traduction d'une capacité à exprimer singulièrement sa pensée, d'une sorte de non résignation à la tradition, aux aléas de la vie.

À vrai dire, Alaidy fait un large usage de deux types de traits idiolectaux : aphorismes et jeux de mots. Deux traits de style qui - de par leur nature métaphorique, leur valeur moderniste, et la portée à la fois philosophique et ludique qu'ils véhiculent - sont des aspects aussi importants que difficiles à traiter. Tâche assez décourageante pour tout traducteur : quand le dit se réduit pour dire plus! Existerait- il, de ce fait, des cas d'absence d'équivalence dans ce roman riche en instances de codification? Ou bien le traducteur saurait-il apprécier à leur juste valeur les innovations langagières apportées par ce jeune écrivain minimaliste?

\section{Les aphorismes}

41 Dans le roman étudié, le héros et son ami représentent une génération révoltée qui se forge son propre lexique en fonction de la situation vécue. Tous deux emploient certains aphorismes, en plus et en dehors des stéréotypes linguistiques répétés par leur communauté, dans le but de déclarer leur différence et le refus qu'ils manifestent vis-àvis de la pensée courante et traditionnelle. C'est aussi et surtout l'ami Abbas, qui, se faisant le mentor du héros, nous livre sa vision du monde à travers de percutants aphorismes. L'idiolecte de ce personnage, lequel apparait par moments comme un être prétentieux et mécontent, est d'une grande importance. C'est à travers trois niveaux de langage que se manifeste sa volonté de "heurter son entourage». Tout d'abord, on note qu'il use d'un lexique "vulgaire et interjectif », employant volontiers bons mots, injures et expressions argotiques. Ensuite, jeux de mots et aphorismes font le reste.

Ici, la possibilité d'une traduction littérale des aphorismes étant complètement exclue, le traducteur tente de trouver la solution ailleurs. La méthode la plus réussie s'avère être l'équivalence. Et la traduction que nous offre Khaled Osman des aphorismes

Traduire, 226 | 2012 
contenus dans le texte de Alaidy, répond, dans la plupart des cas, aux principes de base de toute équivalence: présenter la même signification et la même dénotation, appartenir à la même situation communicative et contenir la même information.

Dans certains cas, c'est la préservation de l'élément pivot de l'aphorisme ou du moins du même champ sémantique qui a permis au traducteur d'appliquer la même stratégie que l'auteur de l'original et de restituer le même effet de styles

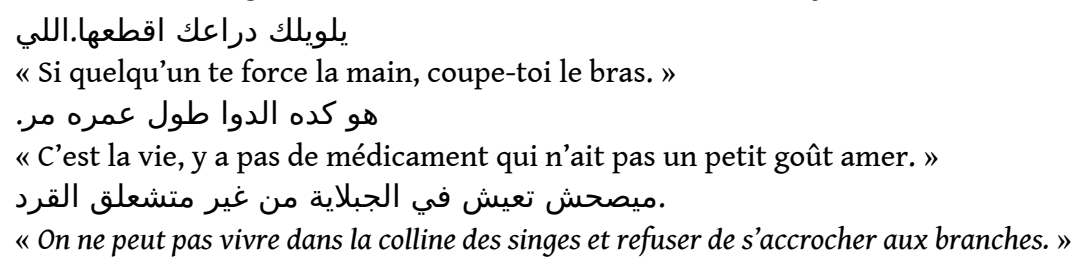

Par ailleurs, l'ami du héros ne manque pas de recourir aux proverbes, qu'ils soient ou non introduits dans le discours par les procédés d'intégration usuels, dans le but de donner plus de crédibilité à ses propos. Il tend même parfois à modifier la structure de certains proverbes en y mettant un peu du sien pour l'adapter à un nouveau contexte. Ceci facilite, en quelque sorte, le repérage de ce qu'on appelle en parémiologie, les moules morphologiques. Le traducteur essaye de se conformer autant que possible à ces modèles et réussit à créer un équivalent qui tient compte à la fois du contenu, de la morphologie et de la fonction symbolique du « faux proverbe » employé dans l'original.

يدي التناكة لولاد المحتاجة.

«Dieu n'a fait plus arrogante que la fille de la mendiante. "

للي له ياقة ما يتضربش علي قفاه.ا

"Si ton col est relevé, tu n'as rien à craindre des taloches sur la nuque."

En considérant les exemples précédents, on s'aperçoit que le traducteur de Alaidy ne se contente pas de garder seulement le contenu sémantique et lexical du proverbe utilisé par l'auteur. Il prend également soin de préserver les caractéristiques formelles, puisque c'est essentiellement par la forme que le proverbe se démarque. Le rythme est, par exemple, un facteur formel de grande importance. Ainsi, rimes et assonances sont intelligemment respectées au moyen de quelques légitimes modulations (au niveau du genre pour le premier exemple : fille à la place de fils pour que le féminin de l'adjectif rime avec le substantif de la fin).

Par ailleurs, au caractère constructif et aux vertus éducatives du langage de l'ami Abbas s'ajoute un caractère artificieux, prouesse verbale et jonglerie avec les mots.

\section{Les jeux de mots}

En effet, tout comme la philosophie, l'humour entretient un rapport privilégié au Minimalisme. Un certain humour se nourrit des décalages et des ruptures de ton propres à cet art. Alaidy recourt essentiellement aux jeux de mots. Ces ressorts ludiques paraissent être un des éléments constitutifs de son système d'écriture. Dans son célèbre ouvrage sur la traduction des jeux de mots, Jacqueline Henry insiste sur l'importance de l'identification de la fonction du jeu de mots au sein du texte à traduire. Selon elle, cette fonction et les effets recherchés sont l'ultime déterminant des choix de traduction. "L'essentiel est que la visée fonctionnelle et pragmatique du jeu de mots soit parfaitement respectée. » (Henry, J. : 2003, 121).

Les exemples répertoriés dans le roman étudié relèvent de ce que Henry appelle «les jeux de mots ponctuels». Elle en dénombre trois: amusement, caractérisation et 
virtuosité. Nous nous intéressons tout particulièrement aux jeux de caractérisation puisque c'est la catégorie rencontrée dans le texte d'Alaidy.

Souvent supprimés ou transférés par simple calque banal du calembour original, les jeux de mots posent un problème majeur à la traduction qui reste, néanmoins, surmontable grâce à une approche analytique précise et correcte. Osman possède un bagage cognitif, des connaissances culturelles relatives au contexte cible qui offrent " une riche source de solutions traductionnelles que le traducteur doit savoir mobiliser à bon escient », comme le souligne Henry (Henry, J. : 2003, 173).

Les solutions qu'il trouve pour faire passer les jeux de mots sont fondées sur une bonne connaissance de la langue du côté de l'encodage comme du côté du décodage. Il fait également preuve d'une grande attention portée aux lieux clés de la signification dans tout le texte, et jouit d'un sens inné de l'humour. Henry précise à ce sujet «qu'il faut une certaine sensibilité aux jeux de mots pour bien les traduire. Cela signifie qu'il faut non seulement les repérer, les comprendre, mais aussi les apprécier pour être à son tour en mesure de produire mieux que de la pure traduction acrobatique. » (Henry, J. : 2003, 258)

Pour mieux apprécier le travail de qualité auquel s'est livré Osman, nous avons passé en revue quelques exemples des plus révélateurs. La plupart des jeux de mots contenus dans le roman d'Alaidy sont formés sur une base purement linguistique (polysémie, homonymie, homophonie, acronymes, etc.).

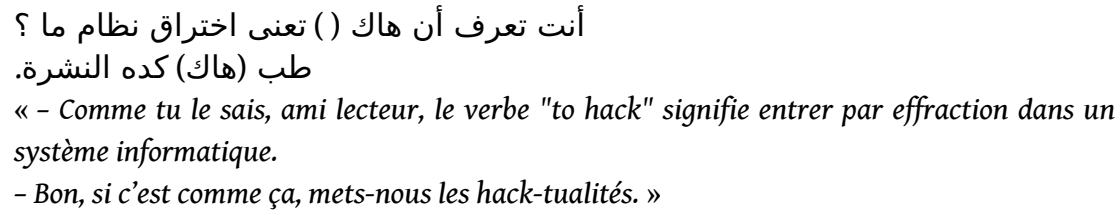

Qu'ils soient homonymiques ou homophoniques, les calembours contenus dans ces phrases sont tous des calembours in praesentia (les deux éléments identiques sont manifestes).

C'est en se servant d'un emprunt qu'Alaidy crée le jeu de mots qu'illustre l'exemple cidessus. Il s'agit là d'un emprunt à l'anglais utilisé dans sa forme originale. Élément majeur du discours, cet emprunt est maintenu en traduction, ainsi que l'image sur laquelle se greffe le jeu. Une égalité presque totale s'installe donc entre le jeu de départ et le jeu d'arrivée réalisée par une sorte de traduction homomorphe exploitant le même procédé mais déplaçant magistralement le calembour d'une partie du discours vers une autre (la première lettre d'un mot à la place de l'impératif d'un verbe). Le comique est assuré par la prononciation sans que la lisibilité et la cohérence du texte ne soient brisées.

54 Le traducteur dispose de différents moyens de rendre les jeux de mots de l'original dans la langue d'arrivée. Il s'est d'ailleurs positionné dans une optique d'équivalence; son objectif n'étant point de produire des correspondances linguistiques mais des équivalences d'effet. Il s'intéresse primordialement au jeu en tant que tel et à l'effet de surprise et d'amusement qu'il engendre.

\section{3) Interlecte : vocabulaire étranger}

Décidément, pour créer un effet de style, Alaidy a plus d'un tour dans son sac. En ce qui concerne les effets basés sur un élément socioculturel, il n'y a pas que la couleur locale qui est exploitée dans ce texte. D'autres procédés servent à la fois de signes identitaires 
et d'indices de classement social ; tel est le cas par exemple du vocabulaire étranger. Pour mieux décrire cette jeunesse américanisée, empruntant son lexique aux moyens de communication et au monde d'Internet et du chat, Alaidy ponctue le discours de ses personnages de mots empruntés essentiellement à l'anglais.

(please), (4 God sake), (not $\mathrm{n}$ the mood), (mix), (package), (volume), (deal), (pull shit), (upgrade), (update), (upload), etc.

Pour faire comprendre au lecteur du texte traduit qu'il s'agissait à la base d'une pratique auctoriale et non d'un simple procédé de traduction, il recourt à un petit détail typographique : la mise en italique de ces anglicismes.

Toutefois, il existe, dans le texte d'Alaidy, un autre type d'emprunts. Il s'agit de termes qui, consacrés par l'usage, figurent dans le texte au moyen d'une transcription phonétique en arabe, non sans subir quelques variations vocaliques chargées, bien évidemment, d'un effet stylistique (montrer le pédantisme d'un personnage, par exemple, ou sa volonté d'appartenir à un monde qui n'est pas le sien). Dans ce cas, pour garder en traduction ces petites déviations phonétiques destinées à noter une prononciation moins distinguée (voire vulgaire) de termes étrangers, le traducteur recourt à certaines astuces orthographiques, comme par exemple: la répétition de certaines consonnes, la transformation d'occlusive bilabiale sourde en occlusive bilabiale sonore $(\mathrm{P} \rightarrow \mathrm{B})$, l'ajout systématique de la voyelle $(\mathrm{i})$ après les consonnes $(\mathrm{t})$, (d) et (b), la transformation de la voyelle antérieure [a] en voyelle postérieure fermée arrondie [o], ou en voyelle antérieure fermée arrondie [ø], ou aussi la confusion entre

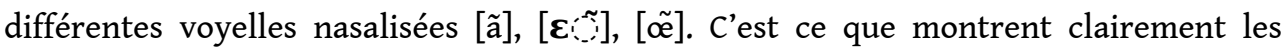
exemples suivants :

Antrreih (أنتريه)Autobile (أوتومبيل)Bebsi (بتر) Betifourr (بتيفور)Bourboires (تبسن) Boyffrainde (بوي فريند) Colculaytor (كولكوليتر) Dedicaycheunz (اونشان) (الديديكيشن)Meetingue

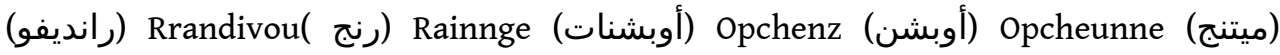
Simbatik (سمبتيك) Souvounir (سوفونير)

Le traducteur fait également suivre la première occurrence de chacun de ces termes dans le texte d'un astérisque, tout en se servant d'une note infrapaginale dans laquelle il explique au lecteur qu'il s'agit de mots anglais ou français intégrés (quelquefois au prix d'une déformation) à l'arabe dialectal.

60 C'est en jouant sur les mêmes types d'incorrections qu'Osman réussit aussi à restituer une technique très spéciale propre à Alaidy: la notation de la prononciation. On rencontre souvent dans son texte des personnages qui, pour prendre de grands airs, prononcent (w) à la place de (b) en arabe,

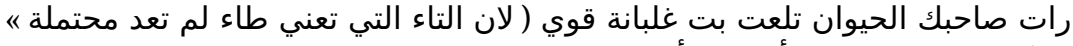

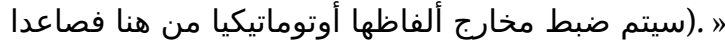

«La femme de ton cochon d'ami s'est "aférée" être une pauvre fille des rues (sa façon de dé former les mots - le f pour le v-étant d'ores et déjà tout à fait insupportable, à par tir de maintenant, la prononciation correcte sera rétablie automatiquement. » (p. 110)

" (متنساش تتوصي بالأكل لحسن أنا بموت في( التبيخ) " ص (19) "

"N’oublie pas de prévoir le manger, parce que j'adore la "guestronomie". » (p. 99)

\section{Le matériel phonographologique}

61 Soucieux de rendre l'originalité du texte en toutes ses parties et de ne rien retrancher au style d'Alaidy, Osman veille scrupuleusement à maintenir l'étrangeté même du 
texte. Toujours dans le but de créer un effet, Alaidy n'hésite pas à combiner, à la manière de la bande dessinée dont il est expert, éléments textuels, visuels et sonores. Le côté auditif est essentiellement exprimé par l'emploi des onomatopées et interjections.

\section{1) Interjections et onomatopées}

Outre l'oralité qui teinte le langage d'Alaidy, son texte se caractérise par une certaine richesse phonique. L'effet sonore y est l'un des éléments de base car, comme dans les bandes dessinées, il est en interaction constante avec les autres éléments : le texte et l'image. C'est un bruitage expressif que nous livre Alaidy; un ajout ponctuel de sons entrant en relation de complémentarité (chose dite + chose entendue) ou d'opposition (chose dite par opposition à chose entendue). Ceci permet un subtil renforcement de l'indirection poétique.

63 En traduction, les onomatopées sont parmi les éléments qui nécessitent un transfert non pas d'un sens ou d'une structure, mais plutôt de l'effet que ceux-ci produisent. De ce fait, la recherche de l'équivalence s'avère être une nécessité, et ceci pour deux raisons bien valables.

Primo, le code sonore n'est pas universel, du moins dans la plupart des cas. Les onomatopées sont, en fait, des unités lexicalisées ayant des valeurs affectives qui diffèrent d'une langue à l'autre; chaque langue ayant sa propre symbolique des sons et ses propres conventions. Comment donc restituer, dans une langue, des créations phonétiques qui appartiennent à une autre langue, si ce n'est à travers l'équivalence?

Secundo, pour l'onomatopée, le rapport qui existe entre signifiant et signifié est un rapport de ressemblance ou d'imitation. C'est essentiellement ce rapport qu'il faut garder en traduction. Sujet très peu traité en traductologie car il s'agit d'un aspect de la bande dessinée qui tend à être survolé sans être abordé en profondeur, la traduction des onomatopées représente une difficulté de taille compte tenu du manque de références en la matière. Entre le français et l'arabe, par exemple, il n'existe à notre connaissance aucun dictionnaire bilingue des onoma- topées. De surcroît, les ouvrages qui traitent du sujet en langue arabe ne s'intéressent malheureusement qu'à la nomenclature des sons, ignorant cet autre aspect fort important de l'onomatopée qu'est la transcription phonétique du bruit lui-même. Dans pareil cas, tout le travail incombe au traducteur qui met à profit ses propres connaissances et les ressources des deux langues en question pour réussir sa traduction.

Une chose est claire : pour Osman, les solutions de facilité sont complètement bannies. Ainsi, d'une manière générale, toutes les onomatopées répertoriées dans l'original arabe ont été conservées en traduction. Aucun sacrifice n'est fait même en cas d'absence d'équivalent dans la langue d'arrivée. De même, le calque n'est pas une option envisageable pour le traducteur. Trois possibilités s'offrent donc à lui :

Adopter des formes propres à la langue française

Il s'agit, à ce moment-là, de trouver, parmi les onomatopées qui figurent dans le dictionnaire, le correspondant employé dans une situation identique dans la langue d'arrivée et reproduisant le même bruit que dans le texte de départ : 


\begin{tabular}{|c|c|c|c|}
\hline L'onomatopée en arabe. & Son contexte. & L'équivalent français. & Sa définition dans le dico. \\
\hline |تيت ت تيت تاا|||تا & $\begin{array}{l}\text { Des klaxons dans un } \\
\text { embouteillage. }\end{array}$ & $\begin{array}{l}\text { Bip, B - Biiippp } \\
\text { Biiii biiiiiiiiiiiiiiip! }\end{array}$ & $\begin{array}{l}\text { n.m. onomatopée. } \\
\text { Signal sonore bref et, } \\
\text { parfois, répété émis par } \\
\text { certains appareils. }\end{array}$ \\
\hline تزررررررن .... تريك. & $\begin{array}{l}\text { Des sonneries de télé- } \\
\text { phone + décrochage de } \\
\text { l'appareil. }\end{array}$ & $\begin{array}{l}\text { Drrrrrinnng } \\
\text { drrrrrrrrrrr...... tic. }\end{array}$ & $\begin{array}{l}\text { Interjection } \\
\text { (onomatopée) } \\
\text { Évoque le bruit d'une son- } \\
\text { nerie. }\end{array}$ \\
\hline 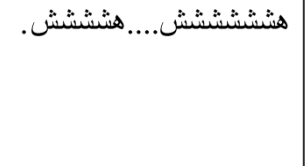 & $\begin{array}{l}\text { Tentatives d'apaisement } \\
\text { d'une personne en larmes. }\end{array}$ & $\begin{array}{l}\text { Chhhhhhhhhhhut } \\
\text { chhhhhhhhhhut. }\end{array}$ & $\begin{array}{l}\text { Interjection } \\
\text { (onomatopée) } \\
\text { Silence. Ne faites pas de } \\
\text { bruit. }\end{array}$ \\
\hline هي هئ. & Des rires. & Hi hi hi. & $\begin{array}{l}\text { Interjection. } \\
\text { Exprime le rire ou les } \\
\text { pleurs. }\end{array}$ \\
\hline
\end{tabular}

Conscient qu'en matière de traduction d'onomatopées, ce qui compte c'est l'effet obtenu, Osman tente de reproduire celui-ci le plus fidèlement possible. Pour ce faire, il applique aux onomatopées dans le texte d'arrivée les mêmes traits produisant par exemple l'effet de durée ou d'intensité du son exprimé (sons forts représentés par des lettres de grande taille, sons persistants décrits par prolongement de la forme, répétition ou doublement de certaines lettres). Il se trouve donc amené à accomplir des prouesses graphiques pour retrouver le même effet que dans l'original, ce qui n'est, $a$ priori, pas du ressort du traducteur.

Aussi, lorsqu'il s'agit de traduire différentes onomatopées reproduisant toutes le bruit d'un coup, par exemple, il convient d'examiner le contexte pour savoir de quel type de coup il s'agit (bruit sec, frapper à la porte, frapper quelqu'un, taper un corps métallique, etc.).

\begin{tabular}{|c|c|c|}
\hline L'onomatopée en arabe. & Son contexte. & L'équivalent français. \\
\hline ديب ... ديب & Une personne rouée de coups. & Pif ! paf! \\
\hline طلك طلك طلك & $\begin{array}{l}\text { Coups sourds sur la carrosserie d'un } \\
\text { minibus. }\end{array}$ & Boum, boum, boum. \\
\hline طا|| & $\begin{array}{l}\text { Coup porté brusquement à un sac en } \\
\text { plastique. }\end{array}$ & Vvvrvvvlaaaaaaaaaaan! \\
\hline
\end{tabular}

La deuxième possibilité consiste à trouver des formes qui ne sont pas propres à la langue française mais déjà intégrées dans celle-ci et répertoriées par les dictionnaires, comme par exemple : slurp (p. 66), choop (p. 50), et hip hip hourra (p. 76), toutes trois empruntées à l'anglais comme l'indiquent les dictionnaires.

La troisième possibilité réside dans la recherche des onomatopées qui ne figurent pas forcément dans les dictionnaires de la langue d'arrivée, mais qui sont consacrées par l'usage courant dans les écrits de cette langue (bandes dessinées ou autre). Tel est le cas de Pschitt (p. 36) et de zit zit zit (p. 47).

Il existe par ailleurs des cas où la langue d'arrivée ne possède pas d'onomatopée consacrée par l'usage qui corresponde au contexte en question. Le traducteur est amené, dans ce cas, à en inventer une. Des modifications sont apportées par Osman 
(changer les lettres ou les combinaisons des lettres) pour remplacer l'onomatopée arabe par une forme plus francisée. Cette adaptation de l'orthographe tient, bien évidemment, compte du type de la sonorité voulue pour ne pas fausser l'effet recherché :

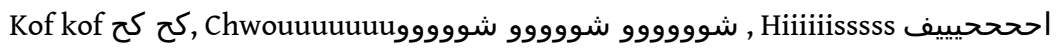

À vrai dire, garder l'onomatopée en traduction ne signifie pas conserver uniquement la richesse sonore du texte, mais également sa portée iconique; puisque ces éléments relèvent aussi bien du visuel que du sonore. Toutefois, il existe dans le texte des éléments exploitant essentiellement le visuel.

\section{2) le visuel et le graphique (les éléments iconographiques)}

75 Les traits iconographiques font réellement partie intégrante du texte d'Alaidy. Il ne s'agit nullement de simples éléments d'illustration visuels mais de vrais signes extralinguistiques auxquels le verbal est intimement lié. Les titres de chapitres sont remplacés par des pictogrammes qui, par référence à leur tracé, sont aussi des idéogrammes traduisant leur contenu. Le traducteur a donc peu de liberté à ce niveau.

Dans le texte d'Alaidy, il ne s'agit pas simplement de traduire ou d'écrire une traduction. Il a fallu veiller à rendre ces dessins transférables. Il s'agit là du seul cas où Khaled Osman a eu recours au calque : le calque de pictogrammes. La taille, la couleur et la forme sont respectées. Même chose pour les émoticônes employées par Alaidy, à la manière des utilisateurs des groupes de discussion, pour indiquer des notes d'humour, mais aussi des émotions.

Alaidy utilise un trait de style qui n'est pas étranger à la logique du Minimalisme : la tension entre mot et syntaxe ; un phénomène qui privilégie le vocable au détriment de la phrase. Certaines marques graphiques contribuent à la représentation esthétique du délire et de l'embarras du personnage, transformant le texte en un espace autre, un espace aux vertiges et aux étrangetés quasi-hallucinatoires.

La retranscription de l'aspect névrotique caractérisant la prononciation du personnage principal se fait, par exemple, à l'aide d'une insistance sur quelques lettres. Alaidy refuse parfois de clore certains mots, les dilatant à l'envi dans l'espace de la page,

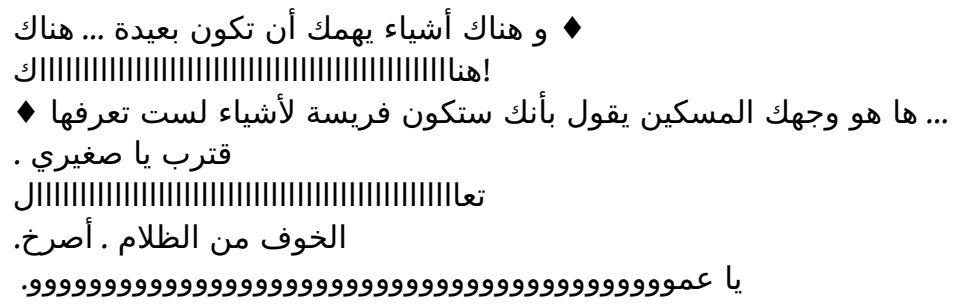

Ce procédé se rencontre aussi dans le texte lorsqu'il s'agit de retranscrire un son quelconque (onomatopées, interjections, effets de rire, altercations et bagarres).

De même, Alaidy n'a de cesse de modifier les caractères des lettres pour susciter maints effets (passage du « je » à une autre voix, création d'un effet de distanciation ironique, indication d'un changement dans l'intonation, mise en relief d'un mot ou d'une idée centrale, etc.). On peut facilement dénombrer dans le texte au moins quatre polices différentes employées, avec variation constante de taille. Sans négliger non plus la mise en italique et en caractères gras. De surcroît, Alaidy use d'une technique très spéciale qui consiste à bouleverser, à des fins esthétiques, le naturel de l'agencement et de l'enchaînement des mots. Fonctionnant essentiellement sur des duos, cette technique 
se présente comme suit : les lettres composant les deux mots sont superposées; elles se referment entre elles comme des parenthèses, de telle manière qu'elles s'entrelacent, refusant de soumettre leurs caractères à l'impératif de juxtaposition du texte imprimé dont dépend la lisibilité du texte classique. Les exemples les plus importants et les plus révélateurs de cet emploi sont, selon nous, ceux qui traduisent des malaises ou des conflits intérieurs du personnage.

81 La poéticité d'un texte ne dépend donc pas seulement de son langage ou de l'agencement de ses idées, mais aussi de sa composition typographique.

Ainsi, c'est à juste titre que Meschonnic souligne que «(...) ce n'est pas de la langue qu'il y a à traduire, mais ce qu'un texte a fait à sa langue, donc il y a à inventer dans la langue d'arrivé e des équivalences de discours : prosodie pour prosodie, métaphore pour métaphore, calembour pour calembour, rythme pour rythme. $»^{4}$

\section{Conclusion}

L'éventail des procédés auxquels Khaled Osman a eu recours pour s'acquitter de sa tâche est très large : une nécessité imposée, certes, par la grande richesse du texte luimême : jeux de mots, langage familier, aphorismes, interjections et onomatopées, etc. C'est E. Nida qui a le mérite d'avoir mis l'accent sur l'importance des connaissances que doit posséder le traducteur sur les us et coutumes d'un peuple pour lui offrir une traduction efficace qui puisse l'atteindre: «presque chaque phrase d'une traduction porte la marque des connaissances anthropologiques du traducteur, car chaque phrase consiste en un ensemble de symboles, relatifs aux types de comportement et de pensée d'une culture, traduit en un autre ensemble de symboles représentant des types différents de compor tement et de pensée. $»(1967: 54)$

L'usage de la méthode d'équivalence dynamique de Nida pour traduire Dans la peau de " Abbas $\mathrm{El}$ 'Abd a certes assuré une bonne migration du sens et du contenu d'un difficile texte littéraire. La traduction qui en résulte a l'avantage de très bien passer en langue française et de transpirer le génie de son créateur. La justesse communicative, la fidélité créative, la reconstitution scrupuleuse de la langue des gens simples de la rue, l'excellent dosage d'un français imagé et coloré qui - en évitant les effets comiques de l'accumulation de marques d'oralité - transmet la verve de l'arabe égyptien, la restitution remarquable des dimensions du sens sans exagération et sans vouer un culte au littéral, et enfin la résolution stratégique du problème de la traduction des formes laconiques forcent l'admiration.

Partant de l'hypothèse qu'une bonne traduction respecte la signification d'ensemble du texte original, Osman ne néglige aucun effet produit par toutes les interactions de mots, de sons et d'images. C'est en se référant à cette méta-signification qu'il réussit sa traduction, se détachant à chaque fois qu'il le faut des mots qui semblent, par endroits, intraduisibles.

Aucune traduction n'est jamais parfaite ou absolue, mais, en somme, celle de Osman fait sans aucun doute partie de celles auxquelles on cherche vainement une faiblesse. Hommage soit donc rendu à ce professionnel, membre de la communauté des passeurs de l'ombre, sans qui notre nouvelle vague de littérature égyptienne serait inaccessible et méconnue. 


\section{BIBLIOGRAPHIE}

ALAIDY Ahmad, 2010, Dans la peau de 'Abbas El ‘Abd, Arles, Actes Sud.

BALLARD Michel, 2001, Oralité et traduction, Arras, Artois Presses Université.

BENJAMIN Walter, 1971, La tâche du traducteur, Paris, Denoël.

BOECKS Cedric, 2006, Linguistic minimalism : origins, concepts, methods and aims, Oxford, Oxford University Press.

CARY Edmond, 1985, Comment faut-il traduire?, Lille, Presses universitaires de Lille.

ECO Umberto, 2007, Dire presque la même chose, Paris, Grasset.

HENRY Jacqueline, 2003, La traduction des jeux de mots, Paris, Presses de la Sorbonne Nouvelle.

HORNSTEIN Norbert, 2005, Understanding Minimalism, Cambridge, Cambridge University Press.

LEDERER Marianne, 1994, La traduction aujourd'hui, Paris, Hachette.

MESCHONNIC Henri, 2007, Éthique et politique du traduire, Paris, Verdier.

MOTTE Warren, 1999, Small worlds : Minimalism in contemporary French literature, London, University of Nebraska Press.

NIDA Eugene, 1964, Toward a science of translating, Leiden, Brill.

NIDA Eugene, 1967, Comment traduire la Bible, Suisse, Alliance biblique universelle.

NIDA Eugene, 1972, Message and mission, California, William Carey Library.

NIDA Eugene, 1978, Coutumes et cultures, Suisse, Edouard Somerville.

NIDA Eugene, TABER Charles, 1969, The theory and practice of translation, Leiden, Brill.

OSEKI-DEPRÉ Inès, 1999, Théorie et pratiques de la traduction littéraire, Paris, Armand Colin.

RICOEUR Paul, 2004, Sur la traduction, Paris, Bayard.

SCHLEIERMACHER Friedrich, 1999, Des différentes méthodes du traduire, Paris, Seuil.

SOLÉ Robert, 2010, En arabe hiéroglyphique, Paris, Le Monde des livres, www.lemonde.fr, consulté le 3 juillet 2011.

\section{NOTES}

1. Meschonnic, H., Éthique et politique du traduire, p. 159.

2. La notion de l'équivalence dynamique a été introduite dans Toward a science of translating, E. J. Brill, Leide, 1964. L'idée est reprise dans plusieurs ouvrages dont le plus important est celui publié en collaboration avec Taber en 1969 sous le titre The theory and practice of translation.

3. Nida, E., Message and mission, New York, Harpers, 1960, p. 192.

4. Meschonnic, H., Op. cit., (p. 74). 


\section{AUTEUR}

\section{SAHAR SAMIR YOUSSEF}

Université Al Azhar, Le Caire

sahar.samiryoussef@gmail.com

Sahar Samir YOUSSEF est maître de conférences au Département de langue française et de traduction, Université Al Azhar du Caire. Elle est titulaire d'un doctorat en linguistique et traduction. Ses travaux sont essentiellement axés sur les problèmes de la traduction littéraire. Traductrice depuis1990, elle a assuré le transfert d'un nombre d'œuvres littéraires françaises en langue arabe. 